\title{
Responsabilidad social universitaria y la calidad de servicio administrativo
}

\section{Social responsibility in the university and quality of administrative service}

Ilich Ivan Pumacayo Palomino, ${ }^{1 a}$ Kriss Melody Calla Vásquez, ${ }^{2}$ Judith Soledad Yangali Vicente, ${ }^{3}$ Melba Rita Vasquez Tomás, ${ }^{4}$ Gissela Karen Arrátia Méndez ${ }^{5}$ y José Luis, Rodríguez López ${ }^{6}$

Universidad Nacional Mayor de San Marcos, Lima, Perú ${ }^{1}$

Universidad Norbert Wiener, Lima, Perú ${ }^{234}$

Universidad Enrique Guzman y Valle, Lima, Perú ${ }^{5}$

Universidad Tecnológica del Perú, Lima, Perú ${ }^{6}$

(iD) Orcid ID: https://orcid.org/0000-0003-1341-2613 ${ }^{1}$

(iD) Orcid ID: https://orcid.org/0000-0003-4976-2332

(iD) Orcid ID: https://orcid.org/0000-0003-0302-5839 3

(iD) Orcid ID: https://orcid.org/0000-0002-1449-3315

(iD) Orcid ID: https://orcid.org/0000-0002-6253-6471

(iD) Orcid ID: https://orcid.org/0000-0002-2573-804X ${ }^{6}$

Recibido: 04 de noviembre 2019

Aceptado: 17 de enero 2020

\section{Resumen}

El presente estudio tuvo como propósito determinar la relación que existe entre la responsabilidad social universitaria y la calidad del servicio administrativo en la universidad. El método fue hipotético deductivo, siendo una investigación básica, transversal correlacional, de diseño no experimental. La muestra trabajada fue probabilística, conformada por 268 estudiantes; utilizandose la encuesta y el cuestionario. El estudio concluyó que existe relación significativa entre la variable responsabilidad social de la universidad y la calidad del servicio administrativo en la universidad; esto implica afirmar 
que: cuanto mayor sea la responsabilidad social de la universidad, mayores serán los niveles de calidad del servicio administrativo.

Palabras clave: responsabilidad social, calidad, servicio administrativo, universidad

\begin{abstract}
This study was aimed to determine the relationship between university social responsibility and quality of administrative services at the university. It was hypothetical deductive method, being a basic, transversal correlational research, non experimental design. The present sample was probabilistic, consisting of 268 students; using the survey and the questionnaire. The study concluded that there is a significant relationship between the variable social responsibility of the university and the quality of the administrative service in the university; This implies stating that: the higher the social responsibility of the university, the higher the levels of quality of the administrative service.
\end{abstract}

Keywords: social responsibility, quality, administrative service, university

\title{
Introducción
}

La responsabilidad social es inherente a la naturaleza y misión de la universidad. Desde una estrategia económica, la responsabilidad social universitaria se basa en la producción de capital humano para la producción de capital social (Melgar, 2015). Asimismo, es necesario aclarar que el papel principal de la universidad es desarrollar las funciones de enseñanza, investigación y responsabilidad social; ello permite crear una visión más amplia de las necesidades en la comunidad generando profesionales competentes y causando impacto positivo en el medio ambiente y la sociedad.

\section{Responsabilidad social universitaria}

Es un concepto que implica un nuevo paradigma, y como tal no está aun claramente definido ni comprendido. Se buscan mejoras adecuadas a la realidad universitaria, mediante autoevaluación y conciencia del potencial para el cambio, inteligencia emocional y diálogo interdisciplinario (Ayala, 2012). En el Perú, la Ley Universitaria No 30220 precisa que: "la responsabilidad social de la universidad es la gestión ética y efectiva (...) a través del 
desempeño de sus funciones: servicios académicos, de investigación y extensión y participación en el desarrollo nacional” (p. 54).

Entre las dimensiones de la responsabilidad social universitaria se encuentra: impactos organizacionales; según Vallaeys (2009), la universidad genera impactos en la vida de su comunidad (personal administrativo, docente y estudiantil), aspectos laborales y de hábitos de vida cotidiana en el campus_ ¿cuáles son los valores que se viven a diario? ¿Cómo se debe vivir en la universidad de forma ciudadana y responsable, en atención a la naturaleza, a dignidad y bienestar de los miembros de la comunidad universitaria?- - y también la contaminación en su entorno (residuos, deforestación, contaminación del aire con el transporte de vehículos, etc.). De esta manera, la universidad deja "huellas" en las personas que conviven en ella y, asimismo, posee "huellas ecológicas" e impactos cognitivos.

Según Vallaeys (2009), la universidad tiene un impacto sobre la sociedad y su desarrollo económico, político, social y cultural mediante los vínculos que establecen con actores externos. Estos impactos reconocen a las universidades como instituciones necesarias en la sociedad. En este sentido, ella tiene un impacto directo sobre la formación de sus profesionales y líderes, y también es un referente y un actor social—¿Qué papel se asume en el desarrollo social, con quién y para qué? ¿Cómo puede la universidad, desde su función específica y experiencia, participar en el progreso social? ¿Qué puede promover (o no) el progreso; vincular (o no) la educación de los estudiantes con la realidad social externa; hacer que el conocimiento esté disponible (o no) para todos?- De esta manera, el entorno social de la universidad reconoce su papel y su capacidad de ser un interlocutor válido para resolver sus respectivos problemas.

\section{Calidad de servicio administrativo}

La calidad de servicio, según Zeithaml y Parasuraman (2004), representa exceder las expectativas y necesidades requeridas en cada uno de los momentos por el cliente o usuario; asimismo, está constituida por todos los atributos o propiedades que la conforman y le otorgan valor. Así, Quispe (2012) precisó que la calidad del servicio es una cultura, una forma de ser, vivir y actuar. La calidad existe cuando los miembros del departamento poseen, comparten y practican una serie de valores, cuyo propósito es satisfacer las necesidades del cliente. Por otro lado, la contribución de Mejías, et al. (2010) señala que "la calidad de servicio es crucial para el éxito de la organización, ya que una alta calidad de servicio brinda 
a las empresas importantes beneficios de productividad, reducción de costos y motivación del personal" (p. 3). Por tanto, de acuerdo a las definiciones de los autores, la calidad de servicio es un factor integral que abarca las diferentes características de calidad, cortesía, oportunidad y rapidez de atención al estudiante; dicha acción no solo sirve para satisfacer las necesidades de los estudiantes, sino ofrecerles un servicio que sea igual o mejor que los demás y que cubra las expectativas con las necesidades en cada servicio que las requiera. Por ende, la calidad de servicio ofrecida es evaluada por los estudiantes en tanto y cuando se sientan satisfecho con lo obtenido.

\section{Dimensiones de la variable calidad de servicio administrativo}

Para abordar este tema de investigación, fue necesario tomar en cuenta lo propuesto por Zeithaml y Parasuraman (2004), quienes dividen la calidad del servicio educativo de la siguiente manera:

(1) Tangibilidad. Segun Zeithaml et al (2006), esto incluye todo tipo de evidencia física del servicio ofrecido: espacio físico, equipamiento-del personal y de los materiales de comunicación; es decir, son los elementos tangibles que se convierten en señales para el estudiante sobre la calidad del servicio educativo. Esto comprende el espacio físico de desarrollo de las distintas actividades universitarias (aulas, laboratorios, instalaciones deportivas, cafeterías, aparcamientos, etc.) e incluye factores como: limpieza, luminosidad, adecuación a los objetivos de uso, etc. De igual forma, la tangibilidad consiste en las percepciones sensoriales relativas al personal académico y administrativo—por ejemplo, la apariencia visual del personal, el contacto con los estudiantes. Por último, también incluye el material de comunicación en soporte físico — desde indicadores de señalización, hasta todo tipo de portafolio informativo sobre la universidad. Por lo tanto, la tangiblidad en la calidad de servicio educativo refiere específicamente a la infraestructura, mobiliario y equipos con que la institución cuenta, así como la higiene y puntualidad en su atención.

(2) Fiabilidad o confiabilidad. Esto se define según Zeithaml, et al (2006), como la habilidad de desarrollar el servicio prometido de forma precisa y fiable. Supone también desempeñar la labor de forma consistente y correctamente. Desde la perspectiva de los estudiantes, una universidad y su personal (académico o administrativo) es fiable, si ofrecen un servicio cumpliendo con lo prometido, tanto a la hora de su prestación, de los atributos 
principales del mismo, como en la resolución de posibles problemas o quejas. Significa incluso que que los profesores y demás empleados de la universidad muestren un interés sincero en resolverles problemas, que realicen su labor correctamente y a tiempo y respondan adecuadamente a los requerimientos de los estudiantes.

(3) Capacidad de respuesta o responsabilidad. Según Zeithaml et al (2006), es la voluntad de ayudar a los clientes en el servicio prestado y facilitarles una pronta respuesta. Esta dimensión pone especial énfasis en el grado de atención así como en la prontitud con la que se atienden las solicitudes de los clientes y sus reclamaciones. Para el estudiante, la capacidad de respuesta de la institución se refleja en la medida en que esta se encuentre siempre disponible para atenderle, con predisposición permanente para prestar su ayuda y responder a sus preguntas, sin tener que esperar prolongadamente para obtenerla. De acuerdo con la acepción anterior, refiere que la respuesta en el servicio educativo ocurre cuando la institución se manifiesta a las necesidades de los estudiantes o padres de una manera rápida, eficaz y amable.

(4) Seguridad. Para Zeithaml et al (2006), la seguridad se debe entender como el conocimiento, cortesía y habilidad de la institución en inspirar buena voluntad y confianza. Así, el personal (académico o administrativo) transmite seguridad a los estudiantes, en la medida en que contaran con el conocimiento apropiado para responder a las preguntas que les hicieran, siendo competentes en el desempeño de su labor y amables en el trato. (p. 119). En este sentido, el estudiante producirá lealtad personal hacia los empleados y los profesores de la universidad, pero también podrá personificar este vínculo con respecto a la propia institución. Incluso es posible que, al menos durante las primeras etapas de la relación con su universidad, el estudiante utilice las evidencias tangibles del servicio para valorar la dimensión de aseguramiento-son ejemplos: las certificaciones de calidad, el posicionamiento en el ranking universitario, los reconocimientos obtenidos, la calidad de publicaciones del departamento, los proyectos de investigación en marcha, y los vínculos con el entorno socio-económico, incluso el orden y la limpieza, entre otros.

(5) Empatía. Para Zeithaml et al (2006), la empatía significa reconocer-mediante una atención personalizada-que cada cliente es único y especial y que sus necesidades son comprendidas y atendidas por la institución (p. 120). En el ámbito de la educación superior, significa que el personal (académico y administrativo) tiene en cuenta los principales 
intereses de los estudiantes, comprende sus necesidades y ofrece a cada uno de ellos una atención adecuada y eficaz. Por ello, la empatía se refiere a las capacidades de la institución educativa para vincularse con sus estudiantes, de manera amigable y respetuosa, resaltando el valor humano y los servicios brindados.

\section{Teorías sobre la calidad del servicio administrativo}

Mariño (2013) afirma que, tradicionalmente, la administración pública ha sido criticada específicamente como prestadora de servicios, y es alrededor de ella que deben formularse los procesos, métodos y procedimientos de la organización. Según Alvarado et al. (2010), la teoría de la calidad en la administración pública es el nivel o grado de respuesta que los empleados y funcionarios ofrecen ante las necesidades de los ciudadanos. Al mismo tiempo, representa el uso adecuado de los medios que permiten mantenerla. La profesionalidad, previsión de los documentos y flexibilidad en proporcionar el servicio hacen efectivo y posible lograr el objetivo principal, que es otro lograr la máxima satisfacción de los usuarios, antes de contratar a funcionarios o empleados de la administración pública. (p. 48).

Por ello, la calidad en la administración pública viene a ser el nivel o el grado de respuesta de los funcionarios o servidores públicos a las demandas y requerimientos del ciudadano-usuario; por tanto, no vincula la calidad con el cliente, sino con la capacidad de los empleados o servidores públicos para dar respuesta a las demandas del ciudadano-usuario. Esto afirma que el estado se preocupe por mejorar la atención al ciudadano en sus instituciones, mediante normas legales que establezcan los principios necesarios para modernizar y mejorar la atención. Ello implica no solo al personal, también al uso de los medios que se dispone y a la revisión permanente de los procesos para satisfacer al usuario cumpliendo los plazos previstos. En resumen, la modernización para la administración pública es la capacidad de una entidad pública de utilizar nuevas tecnologías de información y comunicación para lograr que sus servicios a los ciudadanos sean más accesibles y rápidos, por lo que la calidad es una línea de gestión y la modernización una línea instrumental que apoya la línea de gestión. 


\section{Metodo}

El presente estudio es cuantitativo, por cuanto realizará la medición de las variables y probará hipótesis en base a una muestra, a fin que sus resultados sean generalizados a una determinada población. El método aplicado fue el hipotético-deductivo, ya que se propuso la hipótesis como consecuencia de las inferencias del conjunto de datos empíricos disponibles, cuya relación causa-efecto permitieron llegar al método deductivo (Newman, 2016); esto partió de las evaluaciones muestrales y se establecieron generalizaciones para toda la muestra. Por otro lado, este estudio corresponde al diseño no experimental, transversal correlaciónal. Esto lo convierte en una investigación de carácter descriptivo correlacional, que mide el nivel de relación que existe entre las variables, en el estudio la población fue de 882 personas.

Tabla 1. La población

\begin{tabular}{ll}
\hline Facultad & Población \\
\hline Facultad de Ciencias & 71 \\
Facultad de Ciencias Sociales y Humanidades & 172 \\
Facultad de Tecnología & 150 \\
Facultad de Educación Inicial & 63 \\
Facultad de Pedagogía y Cultura Física & 130 \\
Facultad de Agropecuaria y Nutrición & 120 \\
Facultad de Ciencias Empresariales & 176 \\
TOTAL & 882 \\
\hline
\end{tabular}


Tabla 2. Calculo de la muestra estratificada

\begin{tabular}{lll}
\hline Facultad & Población & Muestra \\
\hline Facultad de Ciencias & 71 & $(0.304 * 71)=22$ \\
Facultad de Ciencias Sociales y Humanidades & 172 & $(0.304 * 172)=52$ \\
Facultad de Tecnología & 150 & $(0.304 * 150)=46$ \\
Facultad de Educación Inicial & 63 & $(0.304 * 63)=19$ \\
Facultad de Pedagogía y Cultura Física & 130 & $(0.304 * 130)=39$ \\
Facultad de Agropecuaria y Nutrición & 120 & $(0.304 * 120)=36$ \\
Facultad de Ciencias Empresariales & 176 & $(0.304 * 176)=54$ \\
TOTAL & 882 & 268
\end{tabular}

El muestreo será de tipo probabilístico, el estudio comprendio 268 docentes.

\section{Resultados}

En la investigación se llegó a los siguientes resultados: la tabla 3 muestra que, de 268 encuestados, el 35,4\% (95) consideran negativa la responsabilidad social universitaria en la universidad, seguido por un $33,2 \%$ (89) quienes la consideran regular; otro $14,6 \%$ (39) la consideran buena; el 8,6\% (23) la consideran deficiente y por último solo un $8.2 \%$ (22) consideran que es eficiente. Si se verifica el promedio $=58,22$ se asume que la responsabilidad social universitaria según los encuestados es mala.

Tabla 3. Distribución de frecuencias de la variable Responsabilidad social universitaria

\begin{tabular}{llll}
\hline Niveles & Rango & Frecuencia Absoluta (f) & Frecuencia Relativa (\%) \\
\hline Muy bueno & {$[85-100]$} & 22 & $8,2 \%$ \\
Bueno & {$[69-84]$} & 39 & $14,6 \%$ \\
Regular & {$[53-68]$} & 89 & $33,2 \%$ \\
Malo & {$[37-52]$} & 95 & $35,4 \%$ \\
Muy malo & {$[20-36]$} & 23 & $8,6 \%$ \\
Total & & 268 & $100,0 \%$ \\
\hline
\end{tabular}

Fuente: Elaboración propia 
La tabla 4 muestran que, de 268 encuestados, el 51,9\% (139) considera regular a los impactos organizacionales en la universidad, seguido por un 17,2\% (46) que la consideran buena; otro 16\% (43) la consideran mala; un 10,1\% (27) la consideran deficiente y por último solo un $4,9 \%$ (13) consideran que es eficiente. Si se verifica el promedio $=15,66$ se asume que los impactos organizacionales según los encuestados, es regular.

Tabla 4. Distribución de frecuencias de la dimensión impactos organizacionales

\begin{tabular}{llll}
\hline Niveles & Rango & Frecuencia Absoluta (f) & Frecuencia Relativa (\%) \\
\hline Muy bueno & {$[22-25]$} & 13 & $4,9 \%$ \\
Bueno & {$[18-21]$} & 46 & $17,2 \%$ \\
Regular & {$[14-17]$} & 139 & $51,9 \%$ \\
Malo & {$[10-13]$} & 43 & $16,0 \%$ \\
Muy malo & {$[5-9]$} & 27 & $10,1 \%$ \\
Total & & 268 & $100,0 \%$ \\
\hline
\end{tabular}

Fuente: Elaboración propia

En la tabla 5 se muestra que, de 268 encuestados, el 26,9\% (72) considera regular los impactos educativos en la universidad, seguido por un 24,6\% (66) quienes los consideran bueno, otro 23,5\% (63) lo consideran malo; un 17,9\% (48) lo consideran deficiente y por último solo un 7,1\% (19) considera que es eficiente. Si se verifica el promedio $=14,47$ se asume que los impactos educativos, según los encuestados es regular.

Tabla 5. Distribución de frecuencias de la dimensión Impactos educativos

\begin{tabular}{llll}
\hline Niveles & Rango & Frecuencia Absoluta (f) & Frecuencia Relativa (\%) \\
\hline Eficiente & {$[22-25]$} & 19 & $7,1 \%$ \\
Bueno & {$[18-21]$} & 66 & $24,6 \%$ \\
Regular & {$[14-17]$} & 72 & $26,9 \%$ \\
Malo & {$[10-13]$} & 63 & $23,5 \%$ \\
Deficiente & {$[5-9]$} & 48 & $17,9 \%$ \\
Total & & 268 & $100,0 \%$ \\
\hline
\end{tabular}

Fuente: Elaboración propia

La tabla 6 muestra que, de 268 encuestados, el 41,4\% (111) consideran malo a los impactos cognitivos en la universidad, seguido por un $23,1 \%$ (62) quienes la consideran regular, 
otro 14,6\% (39) lo consideran bueno, el 13,8\% (37) lo consideran deficiente y por último solo un $7,1 \%$ (19) consideran que es eficiente. Si se verifica el promedio $=13,99$ se asume que los impactos cognitivos, según los encuestados es regular.

Tabla 6. Distribución de frecuencias de la dimensión Impactos cognitivos

\begin{tabular}{llll}
\hline Niveles & Rango & Frecuencia Absoluta (f) & Frecuencia Relativa (\%) \\
\hline Eficiente & {$[22-25]$} & 19 & $7,1 \%$ \\
Bueno & {$[18-21]$} & 39 & $14,6 \%$ \\
Regular & {$[14-17]$} & 62 & $23,1 \%$ \\
Malo & {$[10-13]$} & 111 & $41,4 \%$ \\
Deficiente & {$[5-9]$} & 37 & $13,8 \%$ \\
Total & & 268 & $100,0 \%$ \\
\hline
\end{tabular}

Fuente: Elaboración propia

La tabla 7 muestra que, de 268 encuestados, el 47\% (126) consideran malo a los impactos sociales en la universidad, seguido por un 24,6\% (66) quienes la consideran regular, otro $12,7 \%$ (34) lo consideran bueno, el 8,2\% (22) lo consideran eficiente y por último solo un 7,5\% (20) 
consideran que es deficiente. Si se verifica el promedio $=13,82$ se asume que los impactos sociales, según los encuestados es regular.

Tabla 7. Distribución de frecuencias de la dimensión Impactos sociales

\begin{tabular}{llll}
\hline Niveles & Rango & Frecuencia Absoluta (f) & Frecuencia Relativa (\%) \\
\hline Eficiente & {$[22-25]$} & 22 & $8,2 \%$ \\
Bueno & {$[18-21]$} & 34 & $12,7 \%$ \\
Regular & {$[14-17]$} & 66 & $24,6 \%$ \\
Malo & {$[10-13]$} & 126 & $47,0 \%$ \\
Deficiente & {$[5-9]$} & 20 & $7,5 \%$ \\
Total & & 268 & $100,0 \%$ \\
\hline
\end{tabular}

Fuente: Elaboración propia

En la tabla 8, se puede observar que un 3\% considera la responsabilidad social universitaria como eficiente y también considera eficiente la calidad de servicio administrativo; seguidamente, un 4,9\% considera la responsabilidad social universitaria buena y también considera buena la calidad de servicio administrativo. Por otro lado, un 20,9\% considera la responsabilidad social universitaria regular y también considera regular la calidad de servicio administrativo; asi también el 26,5\% considera la responsabilidad social universitaria como mala y también considera mala la calidad de servicio administrativo; por último, el 6,7\% considera la responsabilidad social universitaria deficiente y también considera deficiente la calidad de servicio administrativo

Tabla 8. Tabla de contingencia responsabilidad social universitaria * Calidad de servicio administrativo 


\begin{tabular}{|c|c|c|c|c|c|c|c|c|c|c|c|c|}
\hline \multirow{3}{*}{$\begin{array}{l}\text { Responsabili } \\
\text { dad social } \\
\text { universitaria }\end{array}$} & \multicolumn{10}{|c|}{ Calidad de servicio administrativo } & \multirow{2}{*}{\multicolumn{2}{|c|}{ Total }} \\
\hline & \multicolumn{2}{|c|}{ Deficiente } & \multicolumn{2}{|c|}{ Malo } & \multicolumn{2}{|c|}{ Regular } & \multicolumn{2}{|c|}{ Bueno } & \multicolumn{2}{|c|}{ Eficiente } & & \\
\hline & $\mathrm{n}$ & $\%$ & $\mathrm{n}$ & $\%$ & $\mathrm{n}$ & $\%$ & $\mathrm{n}$ & $\%$ & $\mathrm{n}$ & $\%$ & $\mathrm{n}$ & $\%$ \\
\hline & & & & & & & & 4,5 & & & & \\
\hline \multirow[t]{2}{*}{ Eficiente } & 2 & $0,7 \%$ & 0 & $0,0 \%$ & 0 & $0,0 \%$ & 12 & $\%$ & 8 & $3,0 \%$ & 22 & $8,2 \%$ \\
\hline & & & & & & & & 4,9 & & & & 14,6 \\
\hline \multirow[t]{2}{*}{ Bueno } & 0 & $0,0 \%$ & 0 & $0,0 \%$ & 8 & $3,0 \%$ & 13 & $\%$ & 18 & $6,7 \%$ & 39 & $\%$ \\
\hline & & & & 12,3 & & 20,9 & & 0,0 & & & & 33,2 \\
\hline \multirow[t]{2}{*}{ Regular } & 0 & $0,0 \%$ & 33 & $\%$ & 56 & $\%$ & 0 & $\%$ & 0 & $0,0 \%$ & 89 & $\%$ \\
\hline & & & & 26,5 & & & & 0,0 & & & & 35,4 \\
\hline \multirow[t]{2}{*}{ Malo } & 18 & $6,7 \%$ & 71 & $\%$ & 6 & $2,2 \%$ & 0 & $\%$ & 0 & $0,0 \%$ & 95 & $\%$ \\
\hline & & & & & & & & 0,0 & & & & \\
\hline \multirow[t]{2}{*}{ Deficiente } & 18 & $6,7 \%$ & 5 & $1,9 \%$ & 0 & $0,0 \%$ & 0 & $\%$ & 0 & $0,0 \%$ & 23 & $8,6 \%$ \\
\hline & & 14,2 & & 40,7 & & 26,1 & & 9,3 & & & & \\
\hline Total & 38 & $\%$ & 109 & $\%$ & 70 & $\%$ & 25 & $\%$ & 26 & $9,7 \%$ & 268 & $100 \%$ \\
\hline \multicolumn{13}{|c|}{ Chi-cuadrado $=76,320 \quad$ g.l. $=16 \quad \mathrm{p}=0.000$} \\
\hline Rho de Spea & & 896 & & & & & & & & & & \\
\hline
\end{tabular}




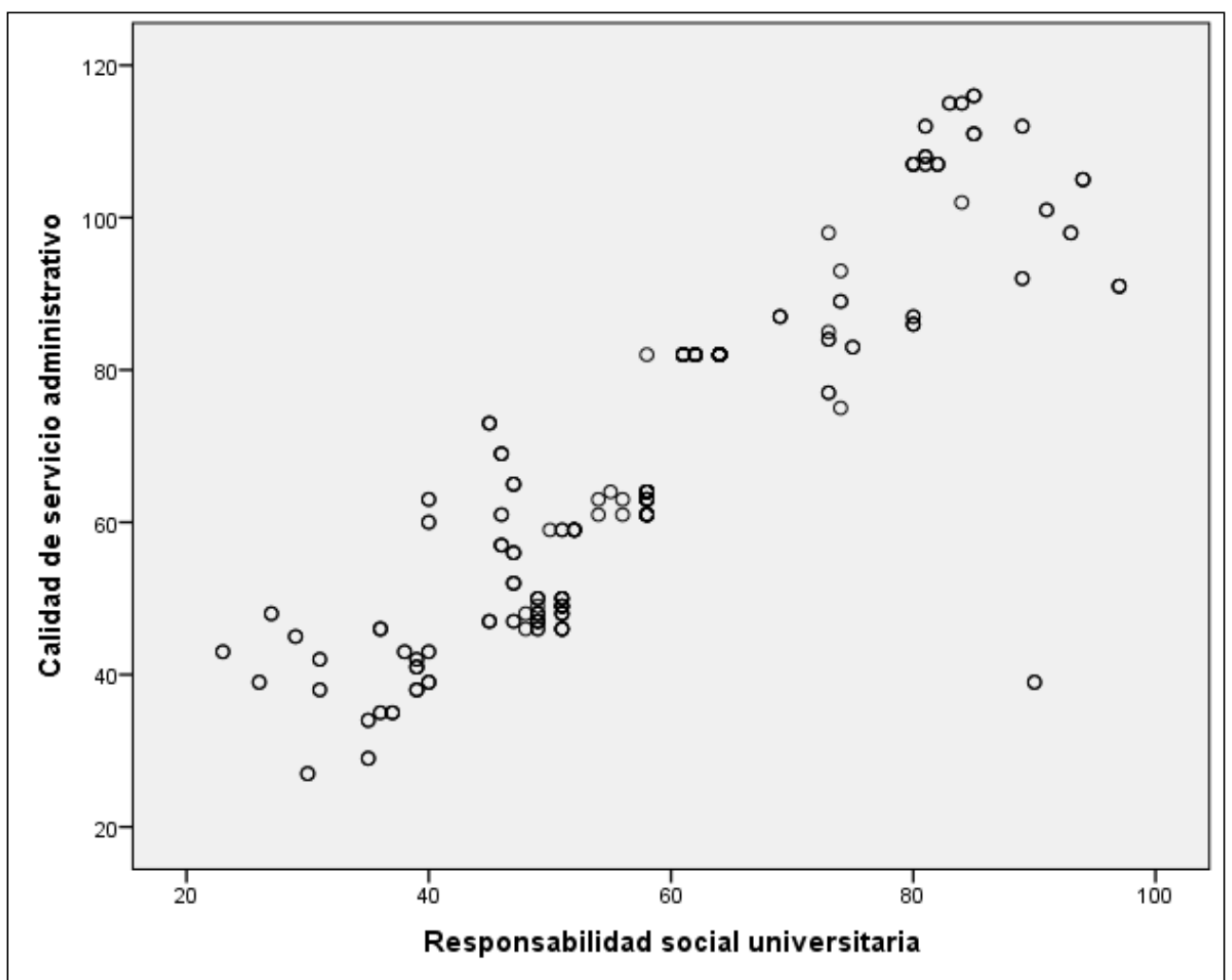

Figura 1. Diagrama de dispersión responsabilidad social universitaria vs Calidad de servicio administrativo

\section{Discusión}

Luego de analizar los resultados, se encontró que existe una relación significativa entre la responsabilidad social de la universidad y la calidad del servicio administrativo en la misma, con un valor significativo de $\mathrm{p}<0.05$ y Spearman Rho $=0.896$ de alta correlación positiva. Por lo tanto, se deduce que cuanto mejor es la responsabilidad social de la universidad, mayor es la calidad del servicio administrativo. Al respecto se hallaron resultados similares en Nuñez (2019), quien manifiesta que se pueden considerar como esenciales para la educación superior las que se refieren a lograr una pertinencia decisiva en el desarrollo social sostenible mediante la formación de valores, compromiso y competencias en todo el personal de la comunidad universitaria y de su entorno transaccional, los mismos que deben satisfacer las necesidades sociales y contribuir a la conservación de la biodiversidad, disminuir el efecto de los desastres naturales y el cambio climático; asi también lograr la equidad, inclusión, la no violencia, reducir la pobreza y fomentar el respeto a los derechos humanos. 
En otro orden, estudios realizados a nivel internacional demuestran la influencia de la responsabilidad social (RSE) en la calidad de servicio; tal es el caso de Saiz (2013), quien afirma que "la RSE es el logro del éxito comercial de modo que se respeten los valores éticos, la gente, las comunidades y el medio ambiente" (p.30). En cuanto a la imagen o marca de la empresa en relación a la responsabilidad social, "existen empresas que son conscientes del impacto que generan sus actividades en la sociedad y aun así no prestan mayor importancia a los aspectos sociales ni ambientales, más allá de las obligaciones que les otorga la Ley” (Bueno, 2019, p.3). En el contexto universitario, es necesario construir una definición precisa que permita conducir la conservación, producción y transmisión del conocimiento científico, la experticia profesional y el pensamiento crítico, hacia la pertinencia social; es decir, la universidad cumple una función social específica que conlleva a pensar, y estar vigilantes, para mejorar los impactos en el mundo empresarial (Vallaeys y Álvarez, 2019).

Algunos resultados también sos similares a los reportados por Eyzaguirre (2016), quién encontró que los alumnos universitario — al comienzo del desarrollo académicopresentaron un nivel moderado de expectativa de desarrollo del servicio académico. Vallaeys (2019) comprobó que las preferencias conceptuales de 740 universitarios latinoamericanos en materia de RSU (Responsabilidad Social universitaria), invitan a articular dos definiciones preferidas por las personas entrevistadas: una centrada en la participación en el desarrollo y otra de la gestión de los impactos universitarios. Sin duda, el involucramiento de la comunidad estudiantil en el cometido de la mejora continua universitaria socialmente responsable, se muestra como un elemento clave para evitar la instrumentalización de la RSU y su puesta al servicio de la "educación bancaria" (Freire, 1970).

\section{Conclusiones}

Existe relación significativa entre la responsabilidad social universitaria y la calidad del servicio administrativo. Por tanto, cuanto mayor sea la responsabilidad social de la universidad, mayores serán los niveles de calidad del servicio administrativo. Asimismo, se evidencia que existe una relación significativa entre la dimensión del impacto educativo de la responsabilidad social universitaria y la calidad del servicio administrativo en la universidad; luego, se puede deducir que cuanto mayor sea la dimensión impactos educativos, habrá niveles más altos en la calidad del servicio administrativo. 
Por otro lado, existe relación entre la dimensión impactos cognitivos y epistemológicos de la responsabilidad social universitaria y la calidad de servicio administrativo en la universidad; esto confirma que, cuanto mayor sea la dimensión impactos cognitivos y epistemológicos, mayor será los niveles en la calidad de servicio administrativo.

Finalmente, existe relación entre la dimensión impactos sociales de la responsabilidad social universitaria y la calidad de servicio administrativo en la universidad; esto indica que, a mayor dimensión de impactos sociales, existirán mayores niveles en la calidad de servicio administrativo.

\section{Referencias}

Alvarado, L. y Hernández, J. (2010). Calidad de servicio en universidades públicas venezolanas. Teacs, núm. 04, pp. 47-50

Ayala, M. (2012). Responsabilidad social universitaria, Recuperado de http://www.redicces.org.sv/jspui/bitstream/10972/91/1/Responsabilidad\%20social\%20 universitaria.pdf> [consulta: noviembre de 2012].

Basantes, R. (2017). Modelo ServQual Académico como factor de desarrollo de la calidad de los servicios educativos y su influencia en la satisfacción de los estudiantes de las carreras profesionales de la Universidad Nacional Chimborazo Riobamba - Ecuador (Tesis doctoral). Lima: Universidad Nacional Mayor de San Marcos. Obtenido de http://cybertesis.unmsm.edu.pe/handle/cybertesis/6132

Bueno, Y. A. (2019). Análisis de la responsabilidad social empresarial en sectores controversiales como herramienta de legitimación vista desde la perspectiva estructuralfuncionalista: caso del sector tabacalero en Colombia (tesis doctoral). Universidad Nacional de Colombia, Bogotá, Colombia. Recuperado de: http://bdigital.unal.edu.co/73569/1/1032379149.2019.pdf

Carrasco, S. (2009). Metodología de investigación científica: Pautas metodológicas para diseñar y elaborar el proyecto de investigación. Lima: Ed. San Marcos.

Chanduví, V. (2013). Orrego, sobre El principio de causalidad en los gastos por prácticas de responsabilidad social universitaria empresarial en el Perú. (Tesis de Maestría). 
Universidad Privada Antenor Orrego, Trujillo, Perú. Recuperado de: http://200.62.226.186/handle/upaorep/396

Eyzaguirre, O. (2016). Expectativa y satisfacción estudiantil por el servicio académico de la Universidad Privada de Tacna en el año 2015. Tacna: Universidad Privada de Tacna. Obtenido de http://repositorio.upt.edu.pe/bitstream/UPT/52/1/eyzaguirre-reinosoomar.pdf

Galviz, G. (2011). Calidad en la Gestión de servicios. Venezuela: Universidad Rafael Urdaneta.

Hernández, R. Fernández C. y Baptista, P. (2014). Metodología de la Investigación Científica. México. McGRAW - Hill Interamericana De México, S.A. de C.V.

Jiménez, M. (2004). Análisis Funcional del Comportamiento y Educación. Hermosillo: Editorial UniSon.

Larrán, M., López, A., Herrera, J. y Andrades, F. (2012). Do spanish public universities use corporate social responsibility as a strategic and differentiating factor? Washington D.C.: International Journal of Humanities and Social Science.

Ley Universitaria $\mathrm{N}^{\circ}$ 30220. Recuperado de: http://www.minedu.gob.pe/reformauniversitaria/pdf/ley_universitaria.pdf

Mariño, A. (2013). Estudio de funciones y actividades del personal administrativo y su incidencia en el proceso de calidad de los servicios de la Facultad de Ingeniería Civil y Mecánica de la Universidad Técnica de Ambato (Tesis de Maestría). Ambato: Universidad Técnica de Ambato. Obtenido de https://repositorio.uta.edu.ec/handle/123456789/5273

Mejías, A., Teixeira, J., Rodríguez, J. y Arzola, M. (2010). Evaluación de la calidad de los servicios universitarios no académicos en una universidad venezolana. Arequipa: 8th Latin American Conference for Engineering and Technology.

Nuñez, I. (2019). Valores, Responsabilidad Social Universitaria (RSU), Educación para el Desarrollo Sostenible (EDS) y Gestión de la Comunicación Organizacional (Tesis de Maestría). Universidad de la Habana.

Ortega, J. (2015). Calidad y satisfacción de los estudiantes europeos de educación superior. Construcción de una escala de medida de la calidad de servicio presencial y electrónico. Salamanca: Universidad De Salamanca. Obtenido de 
https://gredos.usal.es/jspui/bitstream/10366/128480/1/DDOMI_OrtegaMohedanoJ_Cal idadsatisfacci\%C3\%B3nestudiantes.pdf

Palas, L. (2012). La Responsabilidad social universitaria como una forma de mecenazgo empresarial. Propuesta para la creación de una Fundación de la Cámara de Comercio y Producción de Lambayeque. (Tesis de Maestría) Universidad de Piura.

Pereira, M. (2014). Educación superior universitaria: calidad percibida y satisfacción de los egresados (Tesis doctoral). La Coruña: Universidad de la Coruña. Obtenido de http://ruc.udc.es/dspace/bitstream/handle/2183/12349/PereiraPuga_Manuel_TD_2014. pdf?sequence $=2$

Quispe, M. (2012). El modelo administrativo del programa de maestría itinerante de la Escuela de Postgrado de la Universidad Nacional de Educación “Enrique Guzmán y Valle" - Chincha en relación a la calidad de los servicios que reciben los estudiantes (Tesis de Maestría). Lima: Universidad Nacional de Educación Enrique Guzmán y Valle.

Saiz, E. (2013). La responsabilidad social universitaria como atributo diferencial en la imagen corporativa: el caso del sector de la distribución alimentaria en España. (Tesis doctoral) Universidad Pompeu Fabra, de España. Obtenido de https://repositori.upf.edu/handle/10230/22206

Sánchez, H. y Reyes, C. (2006). Metodología y diseño de la investigación científica. Lima: Editorial Visión Universitaria.

Universidad Central de Venezuela (2003). Análisis comparativo de los modelos de gestión académica y administrativa de las Universidades Privadas en Venezuela. TOMO I. Recuperado de http://saber.ucv.ve/bitstream/123456789/9417/1/T026800010846-0PUBLICADO_EDGAR_ORTIZ_julio_11-000-000.pdf

Vaca, R., Moreno, M. y Riquel, F. (2007). Análisis de la responsabilidad social corporativa desde tres enfoques: stakeholders, capital intelectual y teoría institucional. España: Grupo de investigación FEDRA.

Vallaeys, F. y Alvarez, J.(2019). Hacia una definición latinoamericana de responsabilidad social. 
Valderrama, S. (2010). Metodología del trabajo universitario. Perú: San Marcos

Vallaeys, F. (2009). Responsabilidad Social Universitaria. Recuperado de: https://durs.cayetano.edu.pe/images/Biblio/MarcoConceptual/QueEsRSU/presentacion delmarcoteoricodelarsu.pdf

Zeithaml, V. y Parasumarán, A. (2004). Serie de conocimientos relevantes: Servicio Calidad. Cambridge, Mass.: Marketing Instituto de Ciencias. 